\title{
Synthesis, Characterize and Antimicrobial Study of New Chalcones and Pyrazole Derivatives from Progesterone
}

\author{
Nisreen Kais Abood ${ }^{1}$, Hiba Hayder Ibraheem ${ }^{2}$ \\ ${ }^{1}$ Department of Chemistry, College of Science, Al-Mustansiriya University, Baghdad, Iraq \\ ${ }^{2}$ Chemistry Division, Department of Applied Science, University of Technology/Baghdad
}

\begin{abstract}
The aim of this work synthesized a new series of chalcones and pyrazole derivatives from the progesterone, the new steroidal synthesized by the claisen-Schmidt condensation reaction, were react progesterone with different aromatic substituted aldehyde in presence sodium hydroxide afforded chalcones derivatives (1-6). Cyclized the chalcone derivatives by reaction with hydrazine hydrate to afforded pyrazole derivatives (7-12). All synthesized compounds were characterized by measurement melting point, FT-IR spectral, Elemental Analysis and some of them by ${ }^{1} \mathrm{H}-\mathrm{NMR}$ spectral and Antimicrobials activities.
\end{abstract}

Keyword: Progesterone, Chalcone, Pyrazole, Antimicrobial, Biological activity.

\section{Introduction}

Progesterone is one of the most important hormones of the steroidal pregnan [1], and play a major part in female pregnancy[2]. Varying responses to transcription activity monitors it[3] also progesterone brain is derived from the steroidogenic endocring gland or from local synthesis by neural cells [4]. Progesterone is asteroid hormone, a steroid is any of the group of natural or synthetic, fatsoluble, organic compound belonging to the class of the lipids and characterized by molecular are four fused ring to tailing 17 carbon atoms: three six carbon ring and one five carbone ring fused to gather and have three dimensional configuration [5]. Chalcone is important group of the natural product for the different synthesis class, chalcone are easy synthesis by the reacting of two aromatic ring [6]. Chalcone have several biological activities such as anti-inflammatory [7], antimicrobial agent [8], antioxidant [9], antiplatelet [10], and anticancer[11, 12]. These activities are larger attributed due to $\beta$ unsaturated ketone moiety, chalcone important their ability to act as an intermediate for synthesis activity heterocyclic compound such as pyrazole derivatives in this work of research. pyrazol ring is a prominent structural motif found in numerous pharmaceutically active compound, pyrazol important biological active such anti-inflammatory, postmenopausal and osteoporosis [13]

\section{Experimental}

The melting points were determined in open capillary tubes on a Gallen Kamp melting point apparatus and were uncorrected.The FT.IR Spectra of prepared derivatives were taken on Shimadzu-2N, FTIR-8400S, Elemental Analysis \%,. ${ }^{1} \mathrm{H}-\mathrm{NMR}$ Spectra of some prepared derivatives were recorded on a Varian-Mercury 300MHZ Spectrometer, d6-DMSO use as a solvent in ${ }^{1} \mathrm{H}-\mathrm{NMR}$ Spectra.
General procedure for preparation of chalcones (1-6)

A solution of progesterone $(0.01 \mathrm{~mol})$ in $30 \mathrm{ml}$ absolute ethanol, (0.02 mol) substituted aromatic aldehyde in presence $40 \% \mathrm{NaOH}$, the mixture stirring for $4 \mathrm{hrs}$. at room temperature and left over night, after that acidification with dilute hydrochloric acid and filtration afforded solid product and recrystallization from ethanol. The properties of compounds (1-6) show in the table 1 .

General procedure for preparation pyrazole derivatives (7-12)

A mixture of steroidal chalcones (1-6) (0.01 mol) with (0.02 mol)hydrazine hydrate in $30 \mathrm{ml}$ of ethanol was refluxed for 5 hrs., after that cooling the formed solid product was collected by filtration washed with ethanol, dried and crystallization from ethanol afforded pyrazole derivatives (7-12), and recrystallization from ethanol. The properties of compounds (7-12) show in the table 1. 


\section{Scheme 1:}

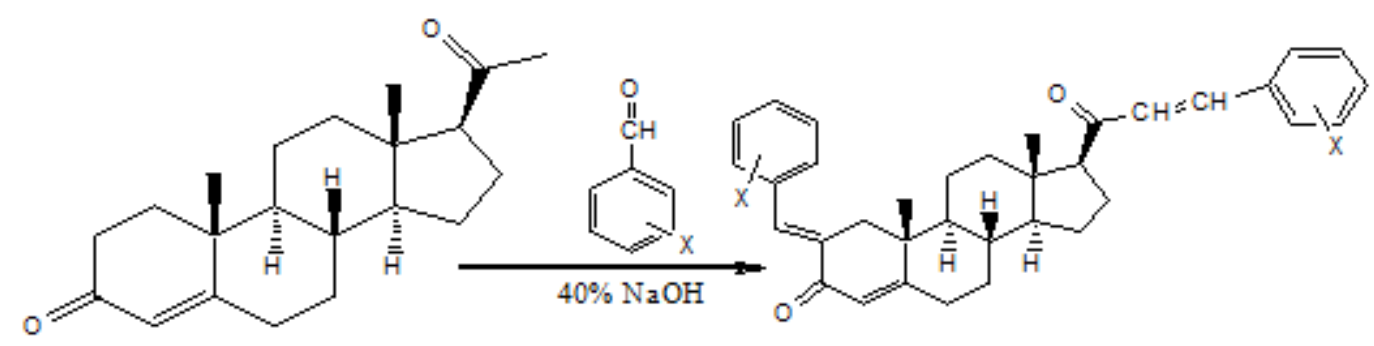

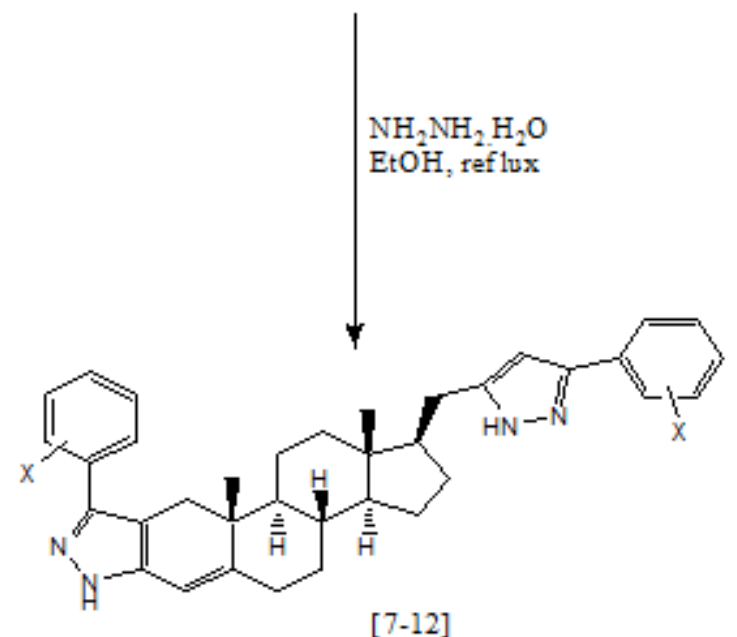

$[7-12]$

$\mathrm{X}: 3-\mathrm{OH}, 4-\mathrm{OCH}_{3}, 2-\mathrm{Br}, 4-\mathrm{NO}_{2}, 2-\mathrm{OH}, 2-\mathrm{NO}_{2}$

\section{In Vitro antimicrobial activity}

The antibacterial activity and antifungal activity was evaluated by the agar well diffusion method [14, 15], the biological activity of the progesterone derivatives were studies chose type of the bacterial which included the Staphylococcus aureus and Streptococcus pneumonia as gram positive and Pseudomonas aeruginosa and E.Coli as gram negative, the antifungal select the $\mathrm{C}$. albicans and $\mathrm{A}$. niger and compared with standard drugs (Ampicillin), in brain hart broth media, which used DMSO as a solvent. This method involves the exposure of the zone of inhibition toward the diffusion of micro-organism on agar plate. The plates were incubated for 24 hrs., $37^{\circ} \mathrm{C}$. and inhibitory zone were recorded.

\section{Result and Discussion}

In this my work research synthesized new derivatives of steroidal chalcone from the reaction progesterone with different substituted aldehyde, scheme 1.

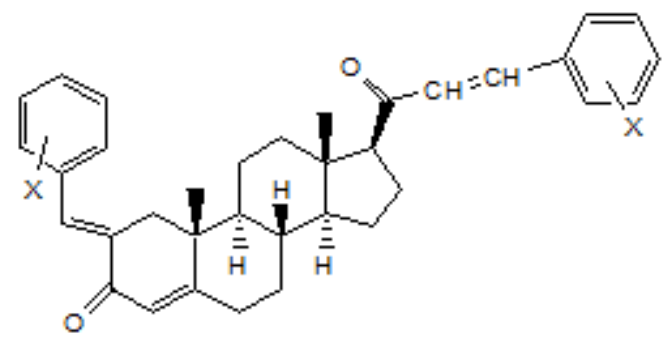

These compounds (1-6) synthesized were confirmed through the physical and spectral data, the FT-IR of compounds (1-6) show the absorption bands of $\mathrm{C}=\mathrm{O}$ at (1747-1665 ) $\mathrm{cm}^{-1}$ respectively, $\mathrm{C}=\mathrm{C}$ aromatic bands stretching at $(1597-1560) \mathrm{cm}^{-1}$ respectively and compounds $(1,5)$ show the bands at $(3401,3410)$ respectively $\mathrm{OH}$ band stretching vibration, compound $(4,6)$ show the asymmetric and symmetric $\left(\mathrm{NO}_{2}\right)$ stretching bands (1566 1357) $\mathrm{cm}^{-1}$ respectively show table 1.Chalcones compounds react with hydrazine hydrate product pyrazole derivatives (7-12).<smiles>[R17]CC[C@]1(C)[C@H](Cc2cc(-c3ccccc3[X])n[nH]2)CC[C@@H]1[C@@H]1CCC2=Cc3[nH]nc(-c4ccccc4[Y])c3C[C@]21C</smiles>

$[7-12]$

The derivatives pyrazole (7-12) show the appearance vNH stretching bands group (3362-3304) $\mathrm{cm}^{-1}, \mathrm{C}=\mathrm{N}$ bands appearance at $(1639-1600) \mathrm{cm}^{-1}$ and $\mathrm{C}=\mathrm{C}$ aromaticbands at (1597-1535) $\mathrm{cm}^{-1},{ }^{1} \mathrm{HNMR}$ (DMSO-d ${ }_{6}$ ) of compound $(7,8$, $9,10)$ show in the table 2.

$[1-6]$ 


\section{International Journal of Science and Research (IJSR) ISSN (Online): 2319-7064 \\ Index Copernicus Value (2013): 6.14 | Impact Factor (2014): 5.611}

Table 1: Physical and spectral data of synthesized compounds:

\begin{tabular}{|c|c|c|c|c|c|}
\hline NO & $\begin{array}{l}\text { Molecular } \\
\text { Formula }\end{array}$ & $\begin{array}{c}\text { Yield } \\
\%\end{array}$ & $\mathrm{Mp} .{ }^{\circ} \mathrm{C}$ & Element analysis calculate / Found \% & $F T-I R \mathrm{~cm}^{-1}$ \\
\hline 1 & $\mathrm{C}_{35} \mathrm{H}_{38} \mathrm{O}_{4}$ & 70 & $220-222$ & C $80.43 / 80.40$ H 7.33/7.30O 12.24/12.20 & $\begin{array}{c}\text { 1712-1672 C=O, } 1560 \mathrm{C}=\mathrm{C}, 3401 \mathrm{OH}, 2970 \mathrm{C}-\mathrm{H} \\
\text { aroma., } 2897 \text { C-H aliph. }\end{array}$ \\
\hline 2 & $\mathrm{C}_{37} \mathrm{H}_{42} \mathrm{O}_{4}$ & 65 & 202-204 & C 80.69/80.65 H 6.93/6.900 11.62/11.60 & $\begin{array}{c}\text { 1730-11710 C=O, } 1570 \mathrm{C}=\mathrm{C}, 3070 \mathrm{C}-\mathrm{H} \text { aroma., } 2890 \\
\text { C-H aliph. }\end{array}$ \\
\hline 3 & $\mathrm{C}_{35} \mathrm{H}_{36} \mathrm{Br}_{2} \mathrm{O}_{2}$ & 65 & 177- 179 & $\begin{array}{c}\text { C } 64.83 / 64.80 \text { H } 5.61 / 5.59 B r 24.64 / 24.6, \text { O } \\
4.93 / 4.90\end{array}$ & $\begin{array}{c}\text { 1701-1689 C=O, } 1597 \text { C=C, } 3061 \mathrm{C}-\mathrm{H} \text { aroma., } 2941 \mathrm{C}- \\
\text { H aliph., } 755 \text { C-Br. }\end{array}$ \\
\hline 4 & $\mathrm{C}_{35} \mathrm{H}_{36} \mathrm{~N}_{2} \mathrm{O}_{6}$ & 75 & $200 d$ & $\begin{array}{c}\text { C72.39/72.35H 6.25/6.20N } \\
4.8 / 4.5016 .53 / 16.5\end{array}$ & $\begin{array}{c}1747-1676 \mathrm{C}=\mathrm{O}, 1589 \mathrm{C}=\mathrm{C}, 3053 \mathrm{C}-\text { Haroma., } 2856 \mathrm{C}- \\
\text { H aliph., } 1566 \mathrm{NO}_{2} \text { asymm.1356 } \mathrm{NO}_{2} \text { symm. }\end{array}$ \\
\hline 5 & $\mathrm{C}_{35} \mathrm{H}_{38} \mathrm{O}_{4}$ & 68 & $198-200$ & C 80.43/80.40 H 7.33/7.30O12.24/12.20 & $\begin{aligned} \text { 1720-1705 C=O, } 1580 \mathrm{C}=\mathrm{C}, 3410 \mathrm{OH}, 3040 \mathrm{C}-\mathrm{H} \\
\text { aroma., 2889C-H aliph. }\end{aligned}$ \\
\hline 6 & $\mathrm{C}_{35} \mathrm{H}_{36} \mathrm{~N}_{2} \mathrm{O}_{6}$ & 70 & 147- 149 & $\begin{array}{c}\text { C } 72.39 / 72.35 \text { H } 6.25 / 6.20 \text { N 4.8/4.5, } \\
\text { O16.53/16.5 }\end{array}$ & $\begin{array}{c}1710-1665 \mathrm{C}=\mathrm{O}, 1580 \mathrm{C}=\mathrm{C}, 3086 \mathrm{C}-\mathrm{Haroma} ., 2939 \mathrm{C}- \\
\mathrm{H} \text { aliph., } 1521 \mathrm{NO}_{2} \text { asymm.1357 } \mathrm{NO}_{2} \text { symm. }\end{array}$ \\
\hline 7 & $\mathrm{C}_{36} \mathrm{H}_{40} \mathrm{~N}_{4} \mathrm{O}_{2}$ & 60 & $210-212$ & $\begin{array}{c}\text { C77.11/77.1H 7.19/7.14 N9.99/9.94 O5.71 } \\
/ 5.65\end{array}$ & $\begin{array}{c}3242 \mathrm{NH}, 3401 \mathrm{OH}, 3073 \mathrm{C}-\mathrm{H} \text { aroma., } 2882 \mathrm{C}-\mathrm{H} \\
\text { aliph., } 1620 \mathrm{C}=\mathrm{N}, 1587 \mathrm{C}=\mathrm{C} .\end{array}$ \\
\hline 8 & $\mathrm{C}_{38} \mathrm{H}_{44} \mathrm{~N}_{4} \mathrm{O}_{2}$ & 60 & $197-199$ & $\begin{array}{c}\text { C 77.52/77.46 H 7.537.50 N9.52/9.50, O 5, } \\
45 / 5.40\end{array}$ & $\begin{array}{l}3342 \mathrm{NH}, 3053 \text { C-H aroma., } 2852 \mathrm{C}-\mathrm{H} \text { aliph., } 1618 \\
\text { C=N, } 1597 \text { C=C. }\end{array}$ \\
\hline 9 & $\mathrm{C}_{36} \mathrm{H}_{38} \mathrm{Br}_{2} \mathrm{~N}_{4}$ & 65 & $212-214$ & $\begin{array}{c}\text { C 62.98/62.93 H 5.58/5.50 N8.16/8.12, Br } \\
23.28 / 23.24 \\
\end{array}$ & $\begin{array}{l}3362 \mathrm{NH}, 3030 \mathrm{C}-\mathrm{H} \text { aroma., } 2953 \mathrm{C}-\mathrm{H} \text { aliph., } 1639 \\
\text { C=N, } 1527 \text { C=C, C-Br } 798 .\end{array}$ \\
\hline 10 & $\mathrm{C}_{36} \mathrm{H}_{38} \mathrm{~N}_{6} \mathrm{O}_{4}$ & 70 & $125-127$ & $\begin{array}{c}\text { C69.88/69.84 H6.19/6.15 N13.58/13.54 O } \\
10.34 / 10.30 \\
\end{array}$ & $\begin{array}{l}3296 \mathrm{NH}, 3055 \mathrm{C}-\mathrm{H} \text { aroma., } 2993 \mathrm{C}-\mathrm{H} \text { aliph., } 1602 \\
\mathrm{C}=\mathrm{N}, 1552 \mathrm{C}=\mathrm{C}, 1535 \mathrm{NO}_{2} \text { asymm.1342 } \mathrm{NO}_{2} \text { symm. }\end{array}$ \\
\hline 11 & $\mathrm{C}_{36} \mathrm{H}_{40} \mathrm{~N}_{4} \mathrm{O}_{2}$ & 70 & $235-237$ & $\begin{array}{c}\text { C 77.11/77.1 H 7.19/7.14N 9.99/9.94 O } 5.71 \\
/ 5.65\end{array}$ & $\begin{array}{c}3254 \text { NH, } 3410 \text { OH, } 3053 \text { C-H aroma., } 2982 \mathrm{C}-\mathrm{H} \\
\text { aliph., } 1616 \text { C=N, } 1580 \mathrm{C}=\mathrm{C} .\end{array}$ \\
\hline 12 & $\mathrm{C}_{36} \mathrm{H}_{38} \mathrm{~N}_{6} \mathrm{O}_{4}$ & 65 & $116-118$ & $\begin{array}{c}\text { C 69.88/69.84 H 6.19/6.15 N13.58/13.54 O } \\
10.34 / 10.30 \\
\end{array}$ & $\begin{array}{l}3304 \mathrm{NH}, 3076 \mathrm{C}-\mathrm{H} \text { aroma., } 2939 \mathrm{C}-\mathrm{H} \text { aliph., } 1616 \\
\mathrm{C}=\mathrm{N}, 1593 \mathrm{C}=\mathrm{C}, 1514 \mathrm{NO}_{2} \text { asymm.1329 } \mathrm{NO}_{2} \text { symm. }\end{array}$ \\
\hline
\end{tabular}

Table 2: Chemical Schiff's ${ }^{1} \mathrm{H}-\mathrm{NMR}$ Spectra of compounds (7-10):

\begin{tabular}{|c|c|}
\hline NO & H-NMR (DMS \\
\hline 7 & $\begin{array}{l}11.2\left(\mathrm{~s}, 1 \mathrm{H}, \mathrm{NH} \text { pyrazole), } 9.2(\mathrm{~s}, \mathrm{IH}, \mathrm{OH}), 6.4(\mathrm{~s}, 1 \mathrm{H}, \mathrm{C} \underline{\mathrm{H}}=\text { ethylen }), 1.2\left(\mathrm{t}, 2 \mathrm{H}, \mathrm{CH}_{2^{-}}\right.\right. \\
\mathrm{C}_{2} \text { cyclopentane), } 2.6\left(\mathrm{~d}, 2 \mathrm{H}, \mathrm{CH}-\mathrm{C}_{2}\right. \\
\text { methylen), } 2.1\left(\mathrm{~s}, 1 \mathrm{H}, \mathrm{C} \underline{\mathrm{H}} \text { cyclo hexane, ), } 1.1\left(\mathrm{~s}, 3 \mathrm{H}, \mathrm{CH}_{3} \text { methyl), 8.0-8.5(m, } \mathrm{C} \underline{\mathrm{H}} \text { protons }\right.\right. \\
\text { aromatic ) }\end{array}$ \\
\hline 8 & $\begin{array}{l}\text { 10.3(s, } 1 \mathrm{H}, \mathrm{NHpyrazole}), 3.9\left(\mathrm{~s}, 3 \mathrm{H}, \mathrm{OCH}_{3}\right), 6.7(\mathrm{~s}, 1 \mathrm{H}, \mathrm{CH}=\text { ethylen }), 1.4\left(\mathrm{t}, 2 \mathrm{H}, \mathrm{CH}_{2}-\right. \\
\mathrm{CH}_{2} \text { cyclopentane), 2.6(d, } 2 \mathrm{H}, \mathrm{CH} \mathrm{CH}_{2} \text { methylen), 2.1(s, } 1 \mathrm{H}, \mathrm{C} \underline{\mathrm{H}} \text { cyclo hexane), } 1.46(\mathrm{~s}, 3 \mathrm{H} \text {, } \\
\mathrm{CH}_{3} \text { methyl), 7.0-7.8(m, } \mathrm{CH} \text { protons aromatic ) }\end{array}$ \\
\hline 9 & $\begin{array}{l}11.1(\mathrm{~s}, 1 \mathrm{H}, \mathrm{NH} H y r a z o l e), 6.5\left(\mathrm{~s}, 1 \mathrm{H}, \mathrm{C} \underline{\mathrm{H}=} \text { ethylen), } 1.7\left(\mathrm{t}, 2 \mathrm{H}, \mathrm{CH}_{2}-\mathrm{C}_{2} \mathrm{cyclopentane}\right), 2.5(\mathrm{~d},\right. \\
2 \mathrm{H}, \mathrm{CH}-\mathrm{C}_{2} \\
\text { methylen), } 2.1\left(\mathrm{~s}, 1 \mathrm{H}, \mathrm{C} \underline{\mathrm{H}} \text { cyclo hexane), } 1.04\left(\mathrm{~s}, 3 \mathrm{H}, \mathrm{CH}_{3} \text { methyl), 7.2-7.8(m, CH protons }\right.\right. \\
\text { aromatic ) }\end{array}$ \\
\hline 10 & $\begin{array}{l}10.3(\mathrm{~s}, 1 \mathrm{H}, \mathrm{NHpyrazole}), 6.9(\mathrm{~s}, 1 \mathrm{H}, \mathrm{CH}=\mathrm{C}), 1.2\left(\mathrm{t}, 2 \mathrm{H}, \mathrm{CH}_{2}-\mathrm{CH}_{2} \mathrm{cyclopentane}\right), 2.6(\mathrm{~d}, 2 \mathrm{H}, \\
\mathrm{CH}-\mathrm{C}_{2} \\
\text { methylen), } 2.2\left(\mathrm{~s}, 1 \mathrm{H}, \mathrm{C} \underline{\mathrm{H}} \text { cyclo hexane), } 1.04\left(\mathrm{~s}, 3 \mathrm{H}, \mathrm{CH}_{3} \text { methyl), 7.9-8.1(m, } \underline{\mathrm{H}} \text { protons }\right.\right.\end{array}$ \\
\hline
\end{tabular}

\section{Biological Activity}

The antimicrobial screening data show that the compounds exhibit antimicrobial properties and it is important to note that new derivatives exhibit more inhibitory effect than original molecule clear that zone of inhibition against the gram positive, gram negative and antifungal act as more powerful. The newly synthesized steroidal chalcones and pyrazole derivatives were tested for Staphylococcus aureus and Streptococcus pneumonia as gram positive and Pseudomonas aeruginosa and E.Coli as gram negative, the antifungal select the albicans and $\mathrm{A}$. niger, the solvent use DMSO as a negative control. The gar disc diffusion method was followed in this screening and the Ampicillin was used as standard drug.
Some of the tested show no significant effect against the used antimicrobial, but the compound have electronwithdrawing group show good activity against and some of derivatives show moderate activity against. The result shows in the table 3 . 


\section{International Journal of Science and Research (IJSR) \\ ISSN (Online): 2319-7064}

Index Copernicus Value (2013): 6.14 | Impact Factor (2014): 5.611

Table 3: Antimicrobial activity of Synthesized compounds

\begin{tabular}{|c|c|c|c|c|c|c|}
\hline \multirow{3}{*}{ Comp.(100ng/ml) } & \multicolumn{6}{|c|}{ Zone of Inhibition (mm) } \\
\hline & \multicolumn{2}{|c|}{ Gram positive } & \multicolumn{2}{|c|}{ Gram negative } & \multicolumn{2}{|c|}{ Anti Fungal } \\
\hline & Streptococcus pneumonia & Staphylococcus aureus & $\begin{array}{c}\text { Pseudomonas } \\
\text { aeruginosa }\end{array}$ & E.Coli & C. albicans & A. niger \\
\hline 1 & 19 & 20 & 19 & 20 & 22 & 17 \\
\hline 2 & ---- & 14 & 24 & 17 & 20 & --- \\
\hline 3 & 19 & --- & 15 & 15 & 21 & --- \\
\hline 4 & 20 & 24 & 20 & 20 & 18 & 21 \\
\hline 5 & 30 & 24 & 18 & --- & 20 & --- \\
\hline 6 & 20 & 19 & --- & 20 & 19 & 17 \\
\hline 7 & 18 & 22 & 20 & 15 & --- & 18 \\
\hline 8 & --- & 14 & 15 & 18 & 12 & --- \\
\hline 9 & -- & --- & -- & 24 & --- & 20 \\
\hline 10 & 20 & 24 & 18 & 15 & 20 & 19 \\
\hline 11 & 17 & 19 & 15 & 19 & 15 & 18 \\
\hline 12 & 19 & 20 & 20 & 20 & 19 & 20 \\
\hline Ampicillin (100ng/ml) & 20 & 22 & 20 & 23 & 21 & 20 \\
\hline Solvent control DMSO & --- & --- & $\begin{array}{ll}-- \\
\end{array}$ & --- & --- & --- \\
\hline
\end{tabular}

\section{Conclusions}

In this my work described in this review indicated the synthesis new chalcone and pyrazole derivatives and the resulted in the produced with high Antibacterial and antifungal activity. Thus research also can act as important tool for medical chemists to develop new steroidal compounds possessing heterocyclic moiety that could be better agents in term efficiency and safety.

\section{References}

[1] Rafat M. Mohareb and Hanaa Y. Hana. Acta Pharm (2008), 58: 29-42

[2] Denise Mullery, Chemistry 150, fall (2008).

[3] D.D. Kumbhar, B.Y.Waghamare, G.R.Pathade, and S.K.Pardeshi. Scholar Research Library (2014), 6(1):224-229.

[4] Michaal Schumacher, Rashad Hussain, Nathalie Gago, Jean-paul Oudinet, Claudia Mattem and Abdel M. Ghoumari. Frontiers in Neuroscience (2012), 6.

[5] Schumacher, M., R. Guennoun, F. Robert. Growth Horm IGF Res.(2004), 14: S18-33.

[6] Ferlini C., Disterfand M.and Vaenti P., Cancer Therapy Pharmacol., (2000), 46, 305.

[7] Nehad A. Abdel latif, Manal M. Saeed, Nesreen S. Ahmed, Rasha Z. Batran and Nadia R. El-Mouhty, International Journal of Innovative Research in Science, Engineering and Technology.(2014), 3:8517-8529.

[8] R.Kalirajan, S.U.Sivakumar, S.Jubie, B. Gowramma and B. Suresh. International Journal of ChemTech Research (2009), 1:27-34.

[9] R. N. Gacche, N. A. Dhole, S. G. Kamble, B. P. Bandgar, J. Enzym. Inhib. Med. Chem. (2008), 23, 28-31.

[10] L. M. Zhao, H. S. Jin, L. P. Sun, H. R. Piao, Z. S. Quan, Bioorg. Med. Chem. Lett, (2005), 15, 5027.

[11]M. Goniotaki, S. Hatziantoniou, K. Dimas, M. Wagner, C. Demetzos, J. Pharm. Pharmacol. (2004), $56,1217-1224$
[12]A. Modzelewska, C. Pettit, G. Achanta, N. E. Davidson, P. Huang, S. R. Khan, Bioorg. Med. Chem.(2006), 14, 3491-3495.

[13] Mounika S., Pharmaceutical Chemistry ;(2013)1, 235-251.

[14]Williams D.H., Fleming, Spectroscopic methods in Organic Chemistry $2^{\text {nded }}$ McGraw Hill Company, (1973).

[15] Online edition for students of organic chemistry Lab. courses at the University of Colorado Boulder, Dept. of Chem. and Biochem. (2007), 5P, 421-429 